\title{
THE SECOND MAXIMAL GROUPS WITH RESPECT TO THE SUM OF ELEMENT ORDERS
}

\author{
Marcel Herzog*, Patrizia Longobardi** and Mercede Maj** \\ *School of Mathematical Sciences \\ Tel-Aviv University \\ Ramat-Aviv, Tel-Aviv, Israel \\ **Dipartimento di Matematica \\ Università di Salerno \\ via Giovanni Paolo II, 132, 84084 Fisciano (Salerno), Italy
}

\begin{abstract}
Denote by $G$ a finite group and let $\psi(G)$ denote the sum of element orders in G. In 2009, H.Amiri, S.M.Jafarian Amiri and I.M.Isaacs proved that if $|G|=n$ and $G$ is non-cyclic, then $\psi(G)<\psi\left(C_{n}\right)$, where $C_{n}$ denotes the cyclic group of order $n$. In 2018 we proved that if $G$ is non-cyclic group of order $n$, then $\psi(G) \leq \frac{7}{11} \psi\left(C_{n}\right)$ and equality holds if $n=4 k$ with $(k, 2)=1$ and $G=\left(C_{2} \times C_{2}\right) \times C_{k}$. In this paper we proved that equality holds if and only if $n$ and $G$ are as indicated above. Moreover we proved the following generalization of this result: Theorem 4. Let $q$ be a prime and let $G$ be a non-cyclic group of order $n$, with $q$ being the least prime divisor of $n$. Then $\psi(G) \leq \frac{\left(\left(q^{2}-1\right) q+1\right)(q+1)}{q^{5}+1} \psi\left(C_{n}\right)$, with equality if and only if $n=q^{2} k$ with $(k, q)=1$ and $G=\left(C_{q} \times C_{q}\right) \times C_{k}$. Notice that if $q=2$, then $\frac{\left(\left(q^{2}-1\right) q+1\right)(q+1)}{q^{5}+1}=\frac{7}{11}$.
\end{abstract}

\section{INTRODUCTION}

In this paper all groups are finite. In [1], H. Amiri, S.M. Jafarian Amiri and I.M. Isaacs introduced the following function on groups:

$$
\psi(G)=\sum\{o(x) \mid x \in G\},
$$

where $o(x)$ denotes the order of $x$. Thus $\psi(G)$ denotes the sum of element orders of the finite group $G$. Denoting the cyclic group of order $n$ by $C_{n}$, they proved the following theorem:

This work was supported by the National Group for Algebraic and Geometric Structures, and their Applications (GNSAGA - INDAM), Italy. The first author is grateful to the Department of Mathematics of the University of Salerno for its hospitality and support, while this investigation was carried out.

Typeset by $\mathcal{A} \mathcal{M} \mathcal{S}-\mathrm{T}_{\mathrm{E}} \mathrm{X}$ 
Theorem AAI. If $G$ is a non-cyclic finite group of order $n$, then

$$
\psi(G)<\psi\left(C_{n}\right) .
$$

Thus the group $C_{n}$ is the unique group of order $n$ which attains the maximal value of $\psi(G)$ among groups of order $n$. We shall call the groups $C_{n}$ "maximal" groups.

Starting from this results recently many authors have studied the function $\psi(G)$ and its relations with the structure of $G$ (see for example [2]-[17]). In the papers [8] and [16] M. Amiri and S.M. Jafarian Amiri, and, independently, R. Shen, G. Chen and C. Wu started the investigation of groups with the second largest value of the sum of element orders.

In [12], we determined the exact upper bound for $\psi(G)$ for non-cyclic groups of order $n$. We proved the following theorem:

Theorem 1. If $G$ is a non-cyclic group of order $n$, then

$$
\psi(G) \leq \frac{7}{11} \psi\left(C_{n}\right) .
$$

Moreover, the equality holds if $n=4 k$ with $(k, 2)=1$ and $G=\left(C_{2} \times C_{2}\right) \times C_{k}$.

Groups of order $n$ satisfying $\psi(G)=\frac{7}{11} \psi\left(C_{n}\right)$ will be called "second maximal". Theorem 1 left open the following problem:

Problem. Determine all second maximal groups.

In this paper we present a solution to this problem. We prove

Theorem 2. The group $G$ of order $n$ is second maximal if and only if $n=4 k$ with $(k, 2)=1$ and $G=\left(C_{2} \times C_{2}\right) \times C_{k}$.

Theorem 1 and Theorem 2 imply the following complete result.

Theorem 3. If $G$ is a non-cyclic group of order $n$, then

$$
\psi(G) \leq \frac{7}{11} \psi\left(C_{n}\right) .
$$

Moreover, the equality holds if and only if $n=4 k$ with $(k, 2)=1$ and $G=\left(C_{2} \times C_{2}\right) \times C_{k}$.

Theorem 2 follows from the following more general result. First, we introduce some notation and remarks. If $q$ is a prime, we shall call a group $G$ a " $q^{*}$-group" if $q$ is the least prime divisor of the order of $G$. We also define the following function of the real variable:

$$
f(x)=\frac{\left(\left(x^{2}-1\right) x+1\right)(x+1)}{x^{5}+1} .
$$

This function is strictly decreasing for $x \geq 2$. Indeed, if $x \geq 2$, then

$$
f(x)=\frac{\left(\left(x^{2}-1\right) x+1\right)(x+1)}{x^{5}+1}=\frac{x^{3}-x+1}{x^{4}-x^{3}+x^{2}-x+1}
$$

and

$$
f^{\prime}(x)=\frac{-x\left(x^{3}\left(x^{2}-4\right)+5 x^{2}+(x-2)(3 x-1)\right)}{\left(x^{4}-x^{3}+x^{2}-x+1\right)^{2}}<0 .
$$

Our general result is the following theorem. 
Theorem 4. Let $q$ be a prime and let $G$ be a non-cyclic $q^{*}$-group of order $n$. Then

$$
\psi(G) \leq \frac{\left(\left(q^{2}-1\right) q+1\right)(q+1)}{q^{5}+1} \psi\left(C_{n}\right)=f(q) \psi\left(C_{n}\right)
$$

and the equality holds if and only if $n=q^{2} k$ with $(k, q !)=1$ and

$$
G=\left(C_{q} \times C_{q}\right) \times C_{k} .
$$

Notice that if $q=2$, then $f(q)=\frac{21}{33}=\frac{7}{11}$. Moreover, if $q$ is an odd prime, then $q>2$ and by our previous remark $f(q)<f(2)=\frac{7}{11}$. Hence it follows by Theorem 4 that if $G$ is a $q^{*}$-group for some odd prime $q$, then

$$
\psi(G) \leq f(q) \psi\left(C_{n}\right)<\frac{7}{11} \psi\left(C_{n}\right)
$$

and the group $G$ is not second maximal. Hence the second maximal groups are $2^{*}$-groups $G$ satisfying the identity $\psi(G)=f(2) \psi\left(C_{n}\right)$ and by Theorem 4 these groups are the groups $G=\left(C_{2} \times C_{2}\right) \times C_{k}$ with $n=4 k$ and $(k, 2)=1$. Thus Theorem 2 follows from Theorem 4 .

We shall call $q^{*}$-groups $G$ of order $n$ with $\psi(G)$ satisfying the identity $\psi(G)=f(q) \psi\left(C_{n}\right)$ "second maximal $q^{*}$-groups". If follows by Theorem 4 that the second maximal groups are exactly the second maximal $2^{*}$-groups, which were determined in Theorem 4.

In addition to determining exactly the second maximal groups, Theorem 4 also determines exactly the second maximal $q^{*}$-groups for all primes $q$. This remark will be our last theorem.

Theorem 5. Let $q$ denote a prime. The $q^{*}$-group $G$ of order $n$ is a second maximal $q^{*}$-group if and only if $n=q^{2} k$ with $(k, q !)=1$ and $G=\left(C_{q} \times C_{q}\right) \times C_{k}$.

In order to conclude this paper, we need only to prove Theorem 4 . The proof of Theorem 4 is achieved in two steps. The first step is the following proposition:

Proposition 6. Let $q$ be a prime and let $G$ be a non-cyclic $q^{*}$-group of order $n$. Then

$$
\psi(G) \leq \frac{\left(\left(q^{2}-1\right) q+1\right)(q+1)}{q^{5}+1} \psi\left(C_{n}\right) .
$$

The second, and final step in our proof of Theorem 4 is the following proposition:

Proposition 7. Let $q$ be a prime and let $G$ be a $q^{*}$-group of order $n$. Then

$$
\psi(G)=\frac{\left(\left(q^{2}-1\right) q+1\right)(q+1)}{q^{5}+1} \psi\left(C_{n}\right)
$$

if and only if $n=q^{2} k$ with $(k, q !)=1$ and

$$
G=\left(C_{q} \times C_{q}\right) \times C_{k} .
$$

It is clear that Theorem 4 follows from Propositions 6 and 7 . The proofs of these propositions will be presented in Sections II and III, respectively. 


\section{Proof of Proposition 6.}

Before beginning with the proof, we shall list some preliminary results from the papers [1], [12] and [14].

Lemma 2.1. Here $G$ denotes a finite group, $p, p_{i}$ denote primes and $n, m, \alpha_{i}$ denote positive integer. The following statements hold.

(1) $\left([12]\right.$, Lemma 2.9(1)) $\psi\left(C_{p^{m}}\right)=\frac{p^{2 m+1}+1}{p+1}=\frac{p\left(p^{m}\right)^{2}+1}{p+1}$.

(2) ([12], Lemma 2.2(3)) If $G=A \times B$, where $A, B$ are subgroups of $G$ satisfying $(|A|,|B|)=1$, then $\psi(G)=\psi(A) \psi(B)$.

(3) ([12], Lemma 2.9(2)) If $n=\prod_{i=1}^{i=m} p_{i}^{\alpha_{i}}$, where $p_{i} \neq p_{j}$ for $i \neq j$, then $\psi\left(C_{n}\right)=$ $\prod_{i=1}^{i=m} \psi\left(C_{p_{i}^{\alpha_{i}}}\right)$.

(4) ([12], Proof of Lemma 2.9(2)) $\psi\left(C_{n}\right) \geq \frac{q}{p+1} n^{2}$.

(5) ([1], Corollary B) If $P$ is a cyclic normal Sylow subgroup of $G$ then $\psi(G) \leq$ $\psi(P) \psi(G / P)$, with equality if and only if $P$ is central in $G$.

(6) ([12], Lemma 2.2(5)) Let $G=P \rtimes F$, where $P$ is a cyclic p-group, $|F|>1$, $(p,|F|)=1$ and $Z=C_{F}(P)$. Then

$$
\psi(G)<\psi(P) \psi(F)\left(\frac{\psi(Z)}{\psi(F)}+\frac{|P|}{\psi(P)}\right) .
$$

(7) ([14], Lemma 2.7) Let $G=P \rtimes F$, where $P$ is a cyclic Sylow $p$-subgroup of $G$ and $F$ is a cyclic p-complement. If $\psi(G)$ has the second largest value for groups of order $|G|$, then $C_{F}(P)$ is a maximal subgroup of $F$.

We now begin with the proof of Proposition 6, which is restated below.

Proposition 6. Let $q$ be a prime and let $G$ be a non-cyclic $q^{*}$-group of order $n$. Then

$$
\psi(G) \leq \frac{\left(\left(q^{2}-1\right) q+1\right)(q+1)}{q^{5}+1} \psi\left(C_{n}\right) .
$$

Proof. From now on, we shall assume that $G$ is a non-cyclic $q^{*}$-group of order $n$ satisfying the inequality

$$
\psi(G)>\frac{\left(\left(q^{2}-1\right) q+1\right)(q+1)}{q^{5}+1} \psi\left(C_{n}\right)
$$

and our aim is to reach a contradiction. Let $p$ denote the largest prime divisor of $n$. Our proof is by induction on the size of $p$. Notice that by our assumptions $n \geq q^{2}$.

If $q=2$, then $\psi(G)>f(2) \psi\left(C_{n}\right)=\frac{7}{11} \psi\left(C_{n}\right)$ and since $G$ is non-cyclic, this contradicts Theorem 1. Therefore we may assume that $q>2$.

By Lemma 2.1(4) $\psi\left(C_{n}\right) \geq \frac{q}{p+1} n^{2}$, which implies that

$$
\psi(G)>\frac{\left(\left(q^{2}-1\right) q+1\right)(q+1) q}{\left(q^{5}+1\right)(p+1)} n^{2} .
$$


Consequently, there exists $x \in G$ such that $o(x)>\frac{\left(\left(q^{2}-1\right) q+1\right)(q+1) q}{\left(q^{5}+1\right)(p+1)} n$, which implies that

$$
[G:\langle x\rangle]<\frac{\left(q^{5}+1\right)(p+1)}{\left(\left(q^{2}-1\right) q+1\right)(q+1) q} .
$$

Suppose, first, that $p=q$. Then $n$ is a power of $q$ and by $(*)$

$$
[G:\langle x\rangle]<\frac{q^{5}+1}{\left(\left(q^{2}-1\right) q+1\right) q} .
$$

Since $\left(\left(q^{2}-1\right) q+1\right) q>\left(q^{2}+1\right) q>\frac{q^{5}+1}{q^{2}}$, it follows that $[G:\langle x\rangle]<q^{2}$. Thus $[G:\langle x\rangle]=q$, which implies that

$$
\psi(G) \leq \psi\left(C_{n / q}\right)+\left(\frac{q-1}{q} n\right) \frac{n}{q}=\frac{q(n / q)^{2}+1}{q+1}+\frac{q-1}{q^{2}} n^{2}=\frac{q^{2}+q-1}{q^{2}(q+1)} n^{2}+\frac{1}{q+1} .
$$

We claim that $\psi(G) \leq \frac{\left(\left(q^{2}-1\right) q+1\right)(q+1)}{q^{5}+1} \psi\left(C_{n}\right)$, yielding a contradiction to our assumption. It suffices to prove that

$$
\frac{q^{2}+q-1}{q^{2}(q+1)} n^{2}+\frac{1}{q+1} \leq \frac{\left(\left(q^{2}-1\right) q+1\right)(q+1)}{q^{5}+1} \cdot \frac{q n^{2}+1}{q+1},
$$

or multiplying by $(q+1)\left(q^{5}+1\right) q^{2}$, that

$$
\left(q^{2}+q-1\right)\left(q^{5}+1\right) n^{2}+q^{2}\left(q^{5}+1\right) \leq\left(q^{3}-q+1\right)(q+1) q^{3} n^{2}+\left(q^{3}-q+1\right)(q+1) q^{2} .
$$

Notice that $\left(q^{2}+q-1\right)\left(q^{5}+1\right)=q^{7}+q^{6}-q^{5}+q^{2}+q-1$ and $\left(q^{3}-q+1\right)(q+1) q^{3}=$ $q^{7}+q^{6}-q^{5}+q^{3}$. Hence it suffices to prove that

$$
\left(q^{7}+q^{2}\right)-\left(q^{6}+q^{5}-q^{4}+q^{2}\right) \leq\left(q^{3}-q^{2}-q+1\right) n^{2} .
$$

But $\left(q^{7}+q^{2}\right)-\left(q^{6}+q^{5}-q^{4}+q^{2}\right)=q^{4}\left(q^{3}-q^{2}-q+1\right)$, so it suffices to prove that $q^{4} \leq n^{2}$, which is true since $n \geq q^{2}$. This proves our claim and completes the proof in the case when $p=q$.

So suppose that $p>q$. Since $q>2$, it follows that $p \geq q+2$. We claim that

$$
[G:\langle x\rangle]<\frac{\left(q^{5}+1\right)(p+1)}{\left(\left(q^{2}-1\right) q+1\right)(q+1) q}<p .
$$

We need to prove that $\left(\left(q^{3}-q+1\right)\left(q^{2}+q\right)-\left(q^{5}+1\right)\right) p>q^{5}+1$ or $\left(q^{4}-q^{3}+q-1\right) p>q^{5}+1$. But $p \geq q+2$ and $q \geq 3$, so

$$
\left(q^{4}-q^{3}+q-1\right) p \geq\left(q^{4}-q^{3}+q-1\right)(q+2)>\left(q^{4}-q^{3}\right)(q+2)=q^{5}+q^{4}-2 q^{3}>q^{5}+1,
$$


as required. So our claim is true.

Hence $[G:\langle x\rangle]<p$ and $\langle x\rangle$ contains a cyclic Sylow $p$-subgroup $P$ of $G$. Since $\langle x\rangle \leq$ $N_{G}(P)$ and $[G:\langle x\rangle]<p$, it follows that $P$ is a cyclic normal subgroup of $G$. Now our assumptions and Lemma 2.1(5) imply that

$$
\psi(P) \psi(G / P) \geq \psi(G)>\frac{\left(\left(q^{2}-1\right) q+1\right)(q+1)}{q^{5}+1} \psi\left(C_{|P|}\right) \psi\left(C_{|G / P|}\right) .
$$

Since $P \cong C_{|P|}$, cancellation yields

$$
\psi(G / P)>\frac{\left(\left(q^{2}-1\right) q+1\right)(q+1)}{q^{5}+1} \psi\left(C_{|G / P|}\right)
$$

Since $G / P$ is a $q^{*}$-group and the maximal prime dividing $|G / P|$ is smaller than $p$, our inductive hypothesis implies that $G / P$ is a cyclic group and $G=P \rtimes F$, where $F \cong G / P$ is a cyclic subgroup of $G,|F| \neq 1$ and $(|F|,|P|)=1$. Since $n=|P||F|$, with $P$ and $F$ being cyclic groups of co-prime orders, it follows that $\psi\left(C_{n}\right)=\psi(P) \psi(F)$.

Let $Z=C_{F}(P)$. By Lemma 2.1(6) we have

$$
\psi(G)<\psi(P) \psi(F)\left(\frac{\psi(Z)}{\psi(F)}+\frac{|P|}{\psi(P)}\right)=\left(\frac{\psi(Z)}{\psi(F)}+\frac{|P|}{\psi(P)}\right) \psi\left(C_{n}\right)
$$

Notice that since $P$ is cyclic and $p \geq q+2$, we have

$$
\frac{|P|}{\psi(P)}=\frac{|P|(p+1)}{p|P|^{2}+1}<\frac{p+1}{p|P|} \leq \frac{p+1}{p^{2}}=\frac{1}{p}+\frac{1}{p^{2}} \leq \frac{q+3}{(q+2)^{2}} .
$$

Consider now the other fraction $\frac{\psi(Z)}{\psi(F)}$. If $C_{F}(P)=F$, then $G=P \times F$ is cyclic, a contradiction. So suppose that $C_{F}(P)=Z<F$. Notice that $\psi(F)$ is a product of $\psi(S)$, with $S$ running over all Sylow subgroups of $F$. Since $Z$ is a proper subgroup of $F$, also $\psi(Z)$ is a similar product, and at least one Sylow subgroup of $Z$, say the Sylow $r$-subgroup of $Z$, is properly contained in the Sylow $r$-subgroup of $F$ of order $r^{s} \geq r$. Hence

$$
\frac{\psi(Z)}{\psi(F)} \leq \frac{r^{2(s-1)+1}+1}{r^{2 s+1}+1}
$$

where $r \geq 3$ and $s \geq 1$.

We claim that the inequality

$$
\frac{r^{2(s-1)+1}+1}{r^{2 s+1}+1} \leq \frac{1}{r^{2}-r+1}
$$

is true. Indeed, this inequality is equivalent to the following sequence of inequalities:

$$
r^{2 s+1}+r^{2}-r^{2 s}-r+r^{2(s-1)+1}+1 \leq r^{2 s+1}+1,
$$


$r-r r^{2 s-2}-1+r^{2 s-2} \leq 0, r-1 \leq(r-1) r^{2 s-2}$ and finally $1 \leq r^{2 s-2}$, which is true. Since $r \geq q$, it follows that

$$
\frac{\psi(Z)}{\psi(F)} \leq \frac{1}{q^{2}-q+1}
$$

Thus by $(* *)$

$$
\psi(G) \leq\left(\frac{1}{q^{2}-q+1}+\frac{q+3}{(q+2)^{2}}\right) \psi\left(C_{n}\right)
$$

and we claim that

$$
\frac{1}{q^{2}-q+1}+\frac{q+3}{(q+2)^{2}}<\frac{\left(\left(q^{2}-1\right) q+1\right)(q+1)}{q^{5}+1},
$$

which yields a contradiction to our assumptions. (Notice that this is not true for $q=2$, being equivalent to $341<336$. But $q \geq 3$, and for $q=3$ it is barely true, being equivalent to $4087<4375$.)

Multiplying the above inequality by $q^{3}+1$ and noting that $q^{3}+1=\left(q^{2}-q+1\right)(q+1)$, our claim becomes

$$
q+1+\frac{(q+3)\left(q^{3}+1\right)}{q^{2}+4 q+4}<\frac{\left(q^{3}-q+1\right)(q+1)\left(q^{3}+1\right)}{q^{5}+1}
$$

Now

$q+1+\frac{(q+3)\left(q^{3}+1\right)}{q^{2}+4 q+4}<q+1+\frac{(q+3)(q+1)\left(q^{2}-q+1\right)}{q^{2}+4 q+3}=q+1+q^{2}-q+1=q^{2}+2$

and

$$
q^{2}+2<\frac{\left(q^{3}-q+1\right)\left(q^{4}+q^{3}\right)}{q^{5}+1}
$$

since this inequality is equivalent to $3 q^{5}+q^{2}+2<q^{6}+q^{3}$, which is true as $q \geq 3$. So

$$
q+1+\frac{(q+3)\left(q^{3}+1\right)}{q^{2}+4 q+4}<\frac{\left(q^{3}-q+1\right)\left(q^{4}+q^{3}\right)}{q^{5}+1}<\frac{\left(q^{3}-q+1\right)(q+1)\left(q^{3}+1\right)}{q^{5}+1}
$$

and our claim follows, yielding a contradiction. The proof of Proposition 6 is now complete.

\section{Proof of Proposition 7.}

In this section we shall prove Proposition 7 which is restated below. As previously, $f(x)$ will denote the function $\frac{\left(\left(x^{2}-1\right) x+1\right)(x+1)}{x^{5}+1}$. 
Proposition 7. Let $q$ be a prime and let $G$ be a $q^{*}$-group of order $n$. Then

$$
\psi(G)=\frac{\left(\left(q^{2}-1\right) q+1\right)(q+1)}{q^{5}+1} \psi\left(C_{n}\right)=f(q) \psi\left(C_{n}\right)
$$

if and only if $n=q^{2} k$ with $(k, q !)=1$ and $G=\left(C_{q} \times C_{q}\right) \times C_{k}$.

Proof. First we show that if $G$ is the group $\left(C_{q} \times C_{q}\right) \times C_{k}$ of order $n=q^{2} k$ with $(k, q !)=1$, then the equality $(* * *)$ holds. Indeed, $\psi(G)=\psi\left(C_{q} \times C_{q}\right) \psi\left(C_{k}\right)=\frac{\left(q^{2}-1\right) q+1}{\psi\left(C_{q^{2}}\right)} \psi\left(C_{n}\right)$ and $\psi\left(C_{q^{2}}\right)=\frac{q^{5}+1}{q+1}$, so $(* * *)$ holds, as claimed.

Now we turn to the "only if" part. So suppose that the $q^{*}$-group $G$ of order $n$ satifies the equality $(* * *)$. Since $f(q)<1, G$ is non-cyclic. Our aim is to show that $n=q^{2} k$ with $(k, q !)=1$ and

$$
G=\left(C_{q} \times C_{q}\right) \times C_{k}
$$

Let $p$ be the maximal prime divisor of $n$. Our proof is by induction on the size of $p$.

Suppose, first that $p=q$. Then $n=q^{r}$ and $r \geq 2$. If $r=2$, then $G$ is a non-cyclic group of order $q^{2}$. The group $C_{q} \times C_{q}$ is the only such group and it clearly satisfies both conditions of Proposition 7. If $q=2$ and $n=2^{3}$, then it is easy to see that $Q_{8}$ attains the maximal value of $\psi$ among the non-cyclic groups of order 8 . But $\psi\left(Q_{8}\right)=27<f(2) \psi\left(C_{8}\right)=\frac{7}{11} \cdot 43$, so no non-cyclic group of order 8 satisfies $(* * *)$. So suppose that either $r=3$ and $q>2$ or $r \geq 4$. Then by Theorem 4.4(1) and Lemma 4.2 in [16], the groups $C_{q} \times C_{q^{r-1}}$ and $M_{q^{r}}=\left\langle a, b \mid a^{q^{r}-1}=b^{q}=1, a^{b}=a^{q^{r-2}+1}\right\rangle$ attain the maximal value of $\psi$ among the non-cyclic groups of order $q^{r}$ and

$$
\psi\left(C_{q} \times C_{q^{r-1}}\right)=\psi\left(M_{q^{r}}\right)=\frac{q^{2 r}+q^{3}-q^{2}+1}{q+1} .
$$

We claim that

$$
\frac{q^{2 r}+q^{3}-q^{2}+1}{q+1}<f(q) \psi\left(C_{n}\right)=\left(\frac{q^{4}+q^{3}-q^{2}+1}{q^{5}+1}\right) \cdot\left(\frac{q^{2 r+1}+1}{q+1}\right)
$$

and hence these values for $n$ are impossible. To show this, we need to prove that

$$
\left(q^{2 r}+q^{3}-q^{2}+1\right)\left(q^{5}+1\right)<\left(q^{4}+q^{3}-q^{2}+1\right)\left(q^{2 r+1}+1\right)
$$

or $\left(q^{7}+q^{4}\right)(q-1)<\left(q^{2 r+3}+q^{2 r}\right)(q-1)$ or $q^{4}<q^{2 r}$, which is true, since $r \geq 3$. Thus if $n=q^{r}$ and $G$ satisfies (***), then only the case $n=q^{2}$ and $G=C_{q} \times C_{q}$ is possible, as required.

Suppose, now, that $p>q$. By Lemma 2.1(4) $\psi\left(C_{n}\right) \geq \frac{q}{p+1} n^{2}$, so

$$
\psi(G)=\frac{\left(\left(q^{2}-1\right) q+1\right)(q+1)}{q^{5}+1} \psi\left(C_{n}\right) \geq \frac{\left(q^{4}+q^{3}-q^{2}+1\right) q}{\left(q^{5}+1\right)(p+1)} n^{2} .
$$


Hence there exists $x \in G$ such that

$$
o(x)>\frac{q^{5}+q^{4}-q^{3}+q}{\left(q^{5}+1\right)(p+1)} n \quad \text { and } \quad[G:\langle x\rangle]<\frac{\left(q^{5}+1\right)(p+1)}{q^{5}+q^{4}-q^{3}+q} .
$$

Case A. Suppose that $p$ divides $[G:\langle x\rangle]$.

Then $[G:\langle x\rangle]=p l$ for some positive integer $l$ and

$$
p l<\frac{q^{5}+1}{q^{5}+q^{4}-q^{3}+q}(p+1)<p+1 .
$$

Hence $l=1$ and $[G:\langle x\rangle]=p$, which implies that $\left(q^{5}+q^{4}-q^{3}+q-q^{5}-1\right) p<q^{5}+1$. Hence

$$
p<\frac{q^{5}+1}{q^{4}-q^{3}+q-1}
$$

and we claim that

$$
q^{5}+1<\left(q^{4}-q^{3}+q-1\right)(q+2)=q^{5}+q^{4}-2 q^{3}+q^{2}+q-2
$$

or $2 q^{3}+3<q^{4}+q^{2}+q$, which is true, since $q \geq 2$. Thus $p<q+2$ and since $p>q$, it follows that $p=q+1$. Hence $q=2, p=3$ and in particular $[G:\langle x\rangle]=3$.

Thus

$$
|G|=n=2^{a} 3^{b} \quad \text { with } a, b \geq 1,
$$

and $\langle x\rangle$ contains a Sylow 2-subgroup $Q$ of $G$. Hence $Q$ is cyclic and the Sylow 3-subgroup $P$ of $G$ is normal in $G$. Thus $G=P \rtimes Q$.

Suppose that there exists $y \in G$ satisfying $[G:\langle y\rangle]=2$. Then $\langle y\rangle$ contains $P$, so also $P$ is cyclic. If there exists $z \in G \backslash\langle y\rangle$ also satisfying $[G:\langle z\rangle]=2$, then $\langle y\rangle \cup\{z\} \subseteq C_{G}(P)$ and $P \leq Z(G)$. But then $G=P \times Q$ is cyclic, a contradiction. Hence if $z \in G \backslash\langle y\rangle$, then $o(z) \leq n / 3$ and $\psi(G) \leq \psi\left(C_{n / 2}\right)+\left(\frac{n}{2}\right)\left(\frac{n}{3}\right)$. But this inequality implies, as shown in the proof of Theorem 1 in [12], that $\psi(G)<\frac{7}{11} \psi\left(C_{n}\right)=f(2) \psi\left(C_{n}\right)$, in contradiction to our assumptions.

So we may assume that $o(z) \leq n / 3$ for all $z \in G$. Since $[G:\langle x\rangle]=3$, it follows that

$$
\psi(G) \leq \psi\left(C_{n / 3}\right)+2\left(\frac{n}{3}\right)\left(\frac{n}{3}\right)=\psi\left(C_{2^{a}}\right) \psi\left(C_{3^{b-1}}\right)+2\left(\frac{n}{3}\right)^{2} .
$$

But this inequality implies, as shown in the proof of Theorem 1 in [12], that $\psi(G)<$ $\frac{7}{11} \psi\left(C_{n}\right)=f(2) \psi\left(C_{n}\right)$, in contradiction to our assumptions.

So Case $\mathbf{A}$ is impossible.

Case B. Suppose that $p$ does not divide $[G:\langle x\rangle]$.

Then $\langle x\rangle$ contains a Sylow $p$-subgroup $P$ of $G$. Hence $P$ is cyclic and $\langle x\rangle \leq N_{G}(P)$. Recall that $[G:\langle x\rangle]<\frac{\left(q^{5}+1\right)(p+1)}{q^{5}+q^{4}-q^{3}+q}$. Hence

$$
[G:\langle x\rangle]=\left[G: N_{G}(P)\right]\left[N_{G}(P):\langle x\rangle\right]=(1+h p)\left[N_{G}(P):\langle x\rangle\right]<\frac{\left(q^{5}+1\right)(p+1)}{q^{5}+q^{4}-q^{3}+q}<p+1
$$


for some non-negative integer $h$. It follows that $h=0$ and $P$ is a normal cyclic Sylow $p$-subgroup of $G$. Thus, by Lemma 2.1(5), we have $\psi(G) \leq \psi(P) \psi(G / P)$, with equality if and only if $P$ is central in $G$. But

$$
\psi(G)=\frac{\left(\left(q^{2}-1\right) q+1\right)(q+1)}{q^{5}+1} \psi\left(C_{n}\right)=\frac{\left(\left(q^{2}-1\right) q+1\right)(q+1)}{q^{5}+1} \psi(P) \psi\left(C_{|G / P|}\right)
$$

so it follows that

$$
\psi(G / P) \geq \frac{\left(\left(q^{2}-1\right) q+1\right)(q+1)}{q^{5}+1} \psi\left(C_{|G / P|}\right)
$$

Suppose, first, that $G / P$ is non-cyclic. Since $G / P$ is a $q^{*}$-group, Proposition 6 implies that

$$
\psi(G / P)=\frac{\left(\left(q^{2}-1\right) q+1\right)(q+1)}{q^{5}+1} \psi\left(C_{|G / P|}\right)
$$

and hence $\psi(G)=\psi(P) \psi(G / P)$. It follows by Lemma 2.1(5) thet $P \leq Z(G)$ and $G=$ $P \times K$ for some subgroup $K$ of $G$ which is isomorphic to $G / P$. Since

$$
\psi(K)=\frac{\left(\left(q^{2}-1\right) q+1\right)(q+1)}{q^{5}+1} \psi\left(C_{|K|}\right)
$$

and since $q|| K \mid$ and $p \nmid|K|$, it follows by our inductive assumptions that $K=\left(C_{q} \times C_{q}\right) \times$ $C_{s}$, with $(s, q !)=1$. But then $G=\left(C_{q} \times C_{q}\right) \times C_{s|P|}$ with $(s|P|, q !)=1$, as required.

So suppose, finally, that $G / P$ is cyclic. Then $G=P \rtimes F$, where $P$ is a cyclic $p$-group and $F$ is a cyclic group of order co-prime to $p$. Thus $n=|P||F|$ and $\psi\left(C_{n}\right)=\psi(P) \psi(F)$.

Since $G$ is a $q^{*}$-group satisfying (***), Proposition 6 implies that $\psi(G)$ has the second largest value among groups of order $n$ and by Lemma 2.1(7) $\left[F: C_{F}(P)\right]$ is a prime. If $\left[F: C_{F}(P)\right] \neq q$, then a Sylow $q$-subgroup $Q$ of $G$ is contained in $C_{F}(P)$. Thus $Q$ is cyclic and it centralizes both $P$ and $F$, so $Q \leq Z(G)$. Hence $G=Q \times K$, with $|K|=k, q \nmid k$ and $K$ is non-cyclic. Now

$$
\psi(G)=\psi(Q) \psi(K)=f(q) \psi\left(C_{n}\right)=f(q) \psi\left(C_{k}\right) \psi(Q)
$$

since $Q$ is cyclic. Hence $\psi(K)=f(q) \psi\left(C_{k}\right)$. But since $q \nmid k$, it follows by Proposition 6 that $\psi(K) \leq f(s) \psi\left(C_{k}\right)$ for some $s>q$. As shown in the Introduction, $f(x)$ is a decreasing function, so $f(s)<f(q)$ and we have reached a contradiction.

Hence $\left[F: C_{F}(P)\right]=q$ and $C_{F}(P) \times P$ is a cyclic subgroup of $G$ of index $q$. So there exists $y \in G$ such that $[G:\langle y\rangle]=q$ and $P \leq\langle y\rangle$.

Suppose, first, that $q=2$. Then $[G:\langle y\rangle]=2$ and $P \leq\langle y\rangle$. If there exists $z \in G \backslash\langle y\rangle$ such that $[G:\langle z\rangle]=2$, then $\langle y\rangle \cup\{z\} \subseteq C_{G}(P)$. Hence $P \leq Z(G)$ and $G=P \times F$ is a cyclic group, a contradiction. So if $z \in G \backslash\langle y\rangle$, then $o(z) \leq n / 3$ and

$$
\psi(G) \leq \psi\left(C_{n / 2}\right)+(n / 2)(n / 3) .
$$


If $p=3$, then $n=2^{a} 3^{b}$ with $a$ and $b$ positive integers, and it follows by the proof of Theorem 1 in [12] that

$$
\psi(G)<\frac{7}{11} \psi\left(C_{n}\right)=f(q) \psi\left(C_{n}\right)
$$

in contradiction to our assumptions.

So it remains to deal with two cases: either $q=2$ and $p \geq 5$, or $q \geq 3$. Let $Z=C_{F}(P)$. By Lemma 2.1(6) we have

$$
\psi(G)<\psi(P) \psi(F)\left(\frac{\psi(Z)}{\psi(F)}+\frac{|P|}{\psi(P)}\right)=\left(\frac{\psi(Z)}{\psi(F)}+\frac{|P|}{\psi(P)}\right) \psi\left(C_{n}\right) .
$$

Since $P$ is cyclic, we have

$$
\frac{|P|}{\psi(P)}=\frac{|P|(p+1)}{p|P|^{2}+1}<\frac{p+1}{p|P|} \leq \frac{p+1}{p^{2}}=\frac{1}{p}+\frac{1}{p^{2}} .
$$

Consider now the other fraction $\frac{\psi(Z)}{\psi(F)}$. As shown in the proof of Proposition $6,[F: Z]=q$ implies that

$$
\frac{\psi(Z)}{\psi(F)} \leq \frac{1}{q^{2}-q+1}
$$

If $q=2$ and $p \geq 5$, then $p \geq q+3$. Thus

$$
\frac{|P|}{\psi(P)}<\frac{1}{p}+\frac{1}{p^{2}} \leq \frac{q+4}{(q+3)^{2}}=\frac{6}{25} \quad \text { and } \quad \frac{\psi(Z)}{\psi(F)} \leq \frac{1}{q^{2}-q+1}=\frac{1}{3} .
$$

Hence

$$
\psi(G)<\left(\frac{1}{3}+\frac{6}{25}\right) \psi\left(C_{n}\right)=\frac{43}{75} \psi\left(C_{n}\right)<\frac{7}{11} \psi\left(C_{n}\right)=f(q) \psi\left(C_{n}\right),
$$

which contradicts our assumptions.

If $q>2$, then $q \geq 3$ and $p \geq q+2$. Thus

$$
\frac{|P|}{\psi(P)}<\frac{1}{p}+\frac{1}{p^{2}} \leq \frac{q+3}{(q+2)^{2}} \quad \text { and } \quad \frac{\psi(Z)}{\psi(F)} \leq \frac{1}{q^{2}-q+1} .
$$

Hence

$$
\psi(G)<\left(\frac{1}{q^{2}-q+1}+\frac{q+3}{(q+2)^{2}}\right) \psi\left(C_{n}\right)
$$

and as shown in the proof of Proposition 6, this inequality implies that

$$
\psi(G)<\frac{\left(\left(q^{2}-1\right) q+1\right)(q+1)}{q^{5}+1} \psi\left(C_{n}\right)=f(q) \psi\left(C_{n}\right),
$$

which contradicts our assumptions.

So in Case B $P$ is normal in $G$, and only groups with $G / P$ non-cyclic can satisfy condition $(* * *)$. As shown above, these groups are as required.

The proof of Proposition 7 is now complete. 


\section{REFERENCES}

1. H. Amiri, S.M. Jafarian Amiri, I.M. Isaacs, Sums of element orders in finite groups, Comm. Algebra 37 (2009), 2978-2980.

2. H. Amiri, S.M. Jafarian Amiri, Sums of element orders on finite groups of the same order, J. Algebra Appl. 10 (2) (2011), 187-190.

3. H. Amiri, S.M. Jafarian Amiri, Sum of element orders of maximal subgroups of the symmetric group, Comm. Algebra 40 (2) (2012), 770-778.

4. M. Baniasad Asad, B. Khosravi, A Criterion for Solvability of a Finite Group by the Sum of Element Orders, J. Algebra 516 (2018), 115-124.

5. S.M. Jafarian Amiri, Second maximum sum of element orders on finite nilpotent groups, Comm. Algebra 41 (6) (2013), 2055-2059.

6. S.M. Jafarian Amiri, Maximum sum of element orders of all proper subgroups of PGL $(2, q)$, Bull. Iran. Math. Soc. 39 (3) (2013), 501-505.

7. S.M. Jafarian Amiri, Characterization of $A_{5}$ and $P S L(2,7)$ by sum of element orders, Int. J. Group Theory 2 (2) (2013), 35-39.

8. S.M. Jafarian Amiri, M. Amiri, Second maximum sum of element orders on finite groups, J. Pure Appl. Algebra 218 (3) (2014), 531-539.

9. S.M. Jafarian Amiri, M. Amiri, Sum of the products of the orders of two distinct elements in finite groups, Comm. Algebra 42 (12) (2014), 5319-5328.

10. S.M. Jafarian Amiri, M. Amiri, Characterization of p-groups by sum of the element orders, Publ. Math. Debrecen 86 (1-2) (2015), 31-37.

11. S.M. Jafarian Amiri, M. Amiri, Sum of the Element Orders in Groups with the Square-Free Order, Bull. Malays. Math. Sci. Soc. 40 (2017), 1025-1034.

12. M. Herzog, P. Longobardi, M. Maj, An exact upper bound for sums of element orders in non-cyclic finite groups, J. Pure Appl. Algebra 222 (7) (2018), 1628-1642.

13. M. Herzog, P. Longobardi, M. Maj, Two new criteria for solvability of finite groups in finite groups, J. Algebra 511 (2018), 215-226.

14. M. Herzog, P. Longobardi and M. Maj, Sums of element orders in groups of order $2 m$ with $m$ odd, Comm. Algebra (to appear).

15. Y. Marefat, A. Iranmanesh, A. Tehranian, On the sum of element orders of finite simple groups, J. Algebra Appl. 12 (7) (2013), 135-138.

16. R. Shen, G. Chen and C. Wu, On groups with the second largest value of the sum of element orders, Comm. Algebra 43 (6) (2015), 2618-2631.

17. M. Tărnăuceanu, D.G. Fodor, On the sum of element orders of finite abelian groups, Sci. An. Univ. "A1.I. Cuza" Iasi, Ser. Math. LX (2014), 1-7. 